\title{
Avaliação do escalonamento fractal de alguns solos brasileiros
}

\author{
Miguel Angel Alfaro Soto ${ }^{1}$, Chang Hung Kiang ${ }^{2}$ \& Orencio Monje Vilar ${ }^{3}$
}

\begin{abstract}
Resumo Este trabalho mostra aspectos do sistema organizacional dos poros de alguns solos inalterados brasileiros, sob o ponto de vista da geometria fractal. O comportamento do escalonamento fractal dos poros é avaliado mediante sua distribuição por meio de ensaios de curva de retenção de água no solo. Os resultados confirmaram hipóteses apontadas por outros autores sobre a existência de distribuições multimodais para um mesmo solo. Estes picos modais guardam relação com a existência de um provável escalonamento, que se mostraram compostos por mais de um na faixa do macroporos e um na de mesoporos. Este fato contribui para uma forma peculiar e escalonada da curva de retenção que limita a utilização de modelos baseados na geometria fractal para previsão da retenção de água. Já o modelo "aqui empregado" foi desenvolvido para suprir parcialmente essas limitações e foi testado em diversos solos, mostrando que os dados obtidos pelo modelo se aproximam satisfatoriamente dos dados experimentais, embora a dimensão fractal resultante para cada solo não guarde uma relação definida com sua textura.
\end{abstract}

Palavras-chave: escalonamento fractal, curva de retenção, modelo fractal.

\begin{abstract}
Evaluation of fractal scaling of some brazilian soils. This paper evaluates the behavior of the fractal scaling of the pores by its distribution through soil-water retention curve tests in the soils. The results confirmed the hypotheses pointed for other authors on the existence of multimodal distributions with respect to the same soil. These modal peaks show a likely composite scalling (macropores and mesopores). This fact contributes to the peculiar and scaling form of the soil-water retention curve limits the use of models based on fractal geometry for forecasting of the water retention. The model proposed was developed to overcome partially these limitations and was tested in diverse soils, showing that, curves obtained from the model match to the experimental data, however the resultant fractal dimension in each soil does not keep a defined relation with its texture.
\end{abstract}

Keywords: fractal scaling, soil-water retention curve, fractal model.

INTRODUÇÃO Diversas áreas do conhecimento tem desenvolvido, nestes últimos anos, modelos em que são requeridas informações das propriedades hidráulicas dos solos não saturados. A determinação dessas propriedades, tais como a condutividade hidráulica do solo não saturado e a retenção de água no solo é, ainda, de emprego limitado, oneroso, demorado e de difícil teorização. Frente a essas dificuldades, a previsão dessas propriedades constitui uma alternativa interessante para sua determinação.

Entre os modelos existentes para previsão da retenção da água no solo, a teoria fractal se apresenta como uma alternativa interessante e inovadora. Diversos autores têm proposto modelos com base numa organização fractal da estrutura do solo (Tyler \& Wheatcraft 1990, Gimenez et al. 1997). Isto é possível visto que a mencionada estrutura possui características da geometria fractal como escalonamento (estrutura que obedece a um sistema hierárquico que se manifesta, por exemplo, na organização de tamanhos dos agregados de maior a menor) e auto-semelhança, parecendo idênticas independentemente da escala em que se apresentam. Gimenez et al. (1997) e Pachepsky et al. (2000), entre outros, têm apresentado informação sobre as aplicações da geometria fractal nas previsões tanto das propriedades de retenção de água como da condutividade hidráulica nos solos não saturados. Xu et al. (2002) têm apresentado e discutido alguns aspectos sobre a modelagem da geometria fractal e apresentaram um modelo para a função da retenção de água no solo e previsão da função condutividade hidráulica baseada na estrutura fractal dos poros do solo. No entanto, a maioria destes modelos se fundamenta no escalonamento de certos componentes da estrutura do solo, tais como partículas ou poros que permitem a previsão de num segmento da curva de retenção ou, em alguns casos, restrita a pequenas faixas de sucção devido aos componentes (poros ou partículas) se mostrarem parcialmente fractais devido a mudanças físicas (como mudanças de massa específica em diferentes tamanhos das partículas), mudanças no escalonamento ou, em alguns casos, não se mostrarem fractais.

O uso dos fractais para modelar a retenção de água no solo e as propriedades hidráulicas dos solos não saturados não é novidade em algumas áreas da $\mathrm{Ci}$ ência do Solo. Por outro lado, seu emprego é relativamente limitado em Geologia, Engenharia Geotécnica ou de Petróleo, Hidrologia, entre outros. Sendo uma 
ferramenta com grande potencial de aplicação, o objetivo deste trabalho é investigar algumas questões referentes ao tipo de estruturas fractais dos poros, em solos brasileiros, devido a terem sido observadas características particulares como distribuição de poros bimodais. Outro objetivo é avaliar a possibilidade da previsão da retenção de água, por meio da geometria fractal e do modelo desenvolvido por Alfaro Soto \& Vilar (2006), com o emprego de uma dimensão fractal, baseada na distribuição dos poros, em diversos solos brasileiros.

\section{MÉTODOS TEÓRICOS E EXPERIMENTAIS}

Escalonamento fractal $\mathrm{Na}$ natureza ocorrem superfícies irregulares e fragmentadas, cujas formas são dificilmente representáveis e mensuráveis pelos conceitos de dimensão da geometria tradicional ou Euclidiana caracterizada por números inteiros. A geometria fractal, por sua vez, permite a análise quantitativa dessas figuras com uma dimensão fracionária.

Um conjunto fractal pode ser definido da forma seguinte:

$$
\mathrm{N}_{\mathrm{n}}=\frac{\mathrm{C}}{\mathrm{r}_{\mathrm{n}}^{\mathrm{D}}}
$$

onde $N_{n}$ representa o número de objetos de dimensão linear característica, $C$ uma constante de proporcionalidade, $r_{n}$ a dimensão linear característica e $D$ a dimensão fractal. A mesma equação pode ser expressa por:

$$
D=\frac{\ln \left(N_{n+1} / N_{n}\right)}{\ln \left(r_{n} / r_{n+1}\right)}
$$

onde os subscritos $n$ e $(n+1)$ identificam duas escalas quaisquer do objeto. A dimensão $D$ pode ser um valor inteiro que representaria a dimensão de objetos como pontos, linhas, planos e objetos tridimensionais $(D=0,1,2 \mathrm{e}$ 3 respectivamente) e, nesse caso, equivaleria à geometria Euclidiana. No entanto, a dimensão fractal é um número fracionário. Estes conceitos podem ser aplicados a um objeto quadrado, ou outras formas (Fig. 1).

Na figura 1-a1, têm-se um triângulo eqüilátero de lados unitários no primeiro estágio. No segundo estágio (Fig. 1-a2), três triângulos de lado unitário $r_{1}=1 / 3$ são colocados em cada lado do triângulo original. Neste caso, o perímetro é composto de 12 lados e, portanto, $\mathrm{N}_{1}=12$. Na etapa seguinte (Fig. 1-a3), 11 triângulos com lado igual a $r_{1}=1 / 9$ são colocados em cada lado da figura da primeira etapa. O perímetro após a modificação é composto por 48 lados, sendo, portanto $\mathrm{N}_{2}=48$. Estes dados, na equação (2), fornecem $\mathrm{D}=1,26$, dimensão do fractal denominado Koch island. Observe-se que este fractal possui dimensão que não é uni nem bidimensional e que caracterizam figuras geométricas fora do alcance da geometria euclidiana.

Além deste modelo ou fractal determinístico, existem diversos outros tipos na literatura, cada um para representar alguma geometria da natureza. Um dos fractais utilizado por diversos autores como um modelo

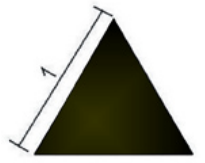

(a1)

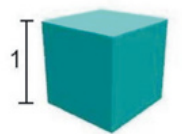

(b1)

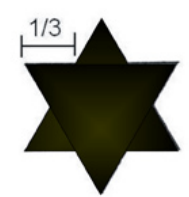

(a2)

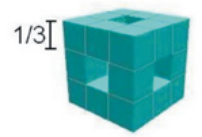

(b2)

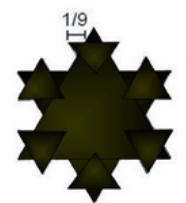

(a3)

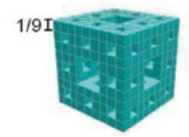

(b3)
Figura 1 - Objetos fractais com diferentes dimensões: a) $D=1,26$; b) $D=2,73$ (Modificado de Turcotte 1992).

de fluxo em meio poroso é a esponja de Menger, que resulta do tapete de Sierpinski estendido para três dimensões, como ilustrado na figura $1 \mathrm{~b}$.

Em objetos naturais, a dimensão fractal é obtida com o auxílio de medições que consistem em quantificar alguma propriedade desse objeto, cuja estrutura possui unidades com formas regulares e diferentes tamanhos característicos $L$. Se a propriedade cumpre as características de hierarquização ou escalonamento fractal (unidades maiores contendo unidades menores e as menores contendo outras menores ainda sucessivamente) e de formas similares em qualquer escala, então é fractal, e o número de unidades, de um tamanho característico $N(L)$, é relacionado com $L$, de acordo com a expressão (3):

$$
\mathrm{N}(\mathrm{L}) \alpha \mathrm{L}^{-\mathrm{D}}
$$

Segundo Giménez et al. (1997), quando se trata de um meio poroso este pode ser separado em diferentes espaços fractais, e a relação (3) pode ser expressa como:

$$
\mathrm{C}_{\mathrm{r}} \alpha \mathrm{r}^{-\mathrm{Dc}}
$$

onde a equação geral é função do raio de um espaço em que o escalonamento fractal se dá para uma determinada característica $C_{r}$, que pode ser a massa $(M)$, superfície $(S)$ ou volume do poro $(V)$. Uma compilação de dimensões obtidas para massa, superfície e volume em diferentes tipos de solos pode ser encontrado em Giménez et al. (1997). Essas dimensões foram mensuradas por meio de diferentes métodos, como da capacidade monocamada, da absorção multicamada, da porosimetria e do box counting, entre outros.

O escalonamento fractal dos poros do solo tem sido utilizado para previsão da retenção de água, bem como da condutividade hidráulica do solo não saturado (Xu et al. 2002, Alfaro Soto \& Vilar 2004a e b). Porém, esta previsão é para solos de baixas faixas de sucção ou umidades próximas da saturação. Ainda, estudos de previsão de curva de retenção em solos brasileiros como o mostrado por Alfaro Soto \& Vilar (2004), suge- 
riram que esses modelos se adéquam a solos com poros de distribuição aparentemente unimodais, o que representa um escalonamento contínuo, pelo menos na faixa de sucção ou umidade utilizada nesses trabalhos.

Infelizmente, tem-se observado que alguns solos inalterados brasileiros podem conter sistemas de poros heterogêneos e complexos, com distribuições de tamanhos de poros multimodais. Segundo Mallants et al. (1997), esses sistemas heterogêneos podem ser resultantes de agregação entre partículas, processos biológicos ou mesmo solifluxão. A esse respeito, Alfaro Soto (2004a) mostrou alguns resultados da característica multimodal de alguns solos do interior de estado de São Paulo, utilizando ensaios de porosimetria por intrusão de mercúrio. A figura 2 mostra um exemplo de distribuição unimodal e bimodal de um caulim compactado e uma areia argilosa inalterada (extraído de Alfaro Soto 2004a).

A heterogeneidade do sistema de poros destes solos é refletida na curva de retenção de água, uma vez que, os tamanhos dos poros estão relacionados com a sucção matricial (decorrente de efeitos capilares e adsorção) do solo, que pode ser mostrada pela teoria do capilar de Buckingham (1907) para um menisco perfeitamente esférico:

$$
\left(\mathrm{u}_{\mathrm{a}}-\mathrm{u}_{\mathrm{w}}\right)=\frac{2 . \sigma_{\mathrm{s}}}{\mathrm{r}}
$$

onde $\left(\mathrm{u}_{\mathrm{a}}-\mathrm{u}_{\mathrm{w}}\right)$ representa a sucção matricial $\left[\mathrm{ML}^{-1} \mathrm{~T}^{-2}\right], r$ o raio do capilar e $\sigma_{\mathrm{s}}$ a tensão superficial originada pela interação das forças intermoleculares produzidas nas zonas de contato entre as partículas do solo, água e ar, sendo esta responsável pela concavidade da interface ar-água e da ascensão da coluna de água no tubo capilar. $O$ valor da tensão superficial é uma característica do líquido e seu valor depende da temperatura, diminuindo à medida que a temperatura aumenta. $\mathrm{O}$ valor da tensão superficial da água a $20^{\circ} \mathrm{C}$ é de $0,07275 \mathrm{~N} / \mathrm{m}$. (Libardi 1995).

Devido à relação existente entre o tamanho dos poros e a sucção, as formas das curvas de retenção são influenciadas pelo escalonamento dos poros. Assim, podem ser obtidas curvas com formas peculiares, com inflexões decorrentes de mudanças na trajetória do escalonamento no sistema de poros. Na figura 3, ilustramse exemplos de curvas de retenção para os mesmos solos da figura 2 (extraído de Alfaro Soto 2004a).

Na figura 3 se observa que o solo areno-argiloso possui uma curva de retenção cuja distribuição de poros é bimodal (segundo mostrado na Fig. 2). Neste caso, peculiarmente a curva apresenta um comportamento assintótico após a segunda inflexão (aproximadamente no valor da umidade residual) mostrando nessa faixa, um valor praticamente constante da umidade com grandes aumentos da sucção, para posteriormente, experimentar um decréscimo da umidade até atingir o valor máximo da sucção. $O$ comportamento assintótico aparentemente se deve, a essa faixa possuir tamanhos de poros relativamente uniformes. Já a Caulinita, mostra uma curva de retenção típica, decor-

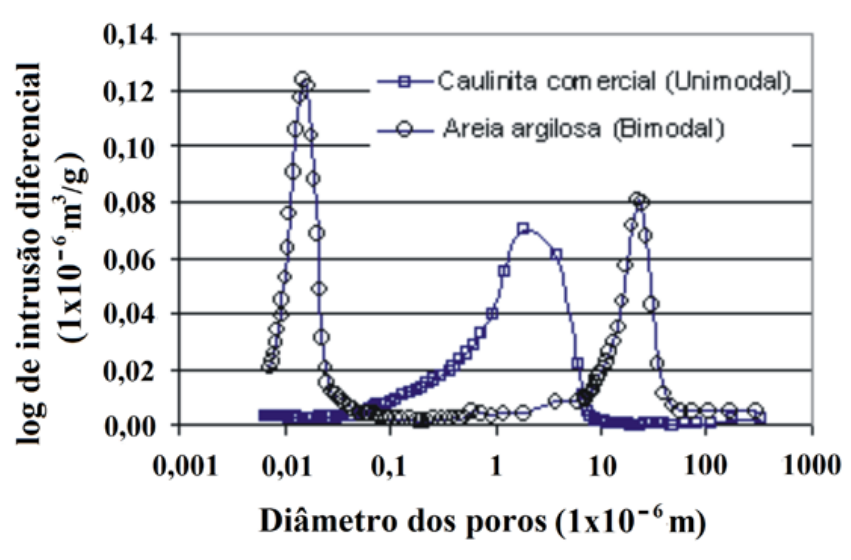

Figura 2 - Distribuição unimodal e bimodal (Alfaro Soto 2004a).

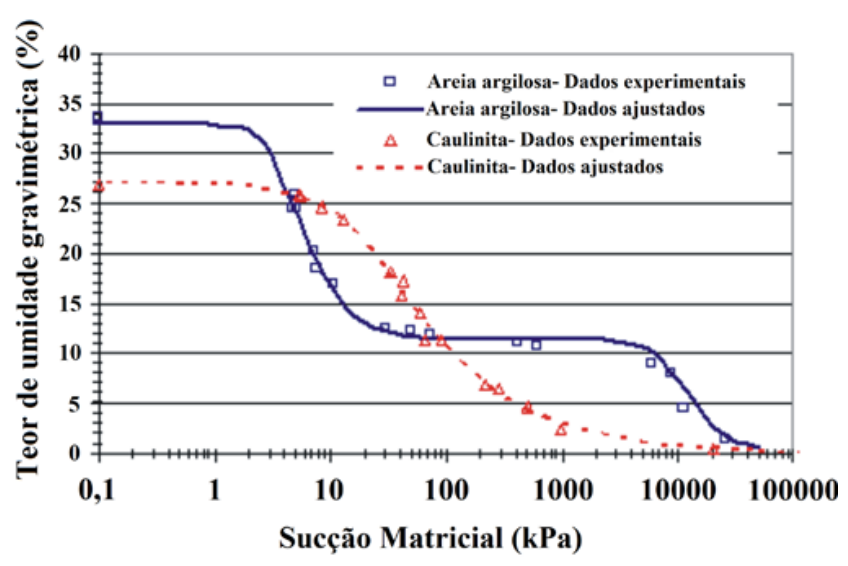

Figura 3 - Curvas de retenção com características de distribuição de poros unimodal e bimodal (Alfaro Soto 2004a).

rente de uma distribuição de poros unimodal (conforme mostrado na Fig. 2), com redução de tamanhos de forma gradual e contínua.

\section{DISTRIBUIÇÃO DOS POROS A PARTIR DA CURVA DE RETENÇÃO DE ÁGUA NO SOLO A} distribuição do tamanho dos poros pode ser determinada derivando a equação que descreve a curva de retenção de água no solo (Stoicescu et al. 1996).

Diversos são os modelos utilizados para ajuste da curva de retenção. Um dos mais empregados e aceitos é o modelo de van Genuchten (1980), que é dado por:

$$
\theta=\theta_{\mathrm{r}}+\frac{\left(\theta_{\mathrm{s}}-\theta_{\mathrm{r}}\right)}{\left(1+(\alpha \psi)^{\mathrm{n}}\right)^{\mathrm{n}}}
$$

Derivando a equação (6), obtém-se:

$$
f(\psi)=\frac{m n \alpha(a \psi)^{n-1}}{\left(1+(\alpha \psi)^{n}\right)^{m+1}}
$$


o nde $f(\psi)$ representa a função de probabilidade que determina a distribuição do tamanho do poro para uma determinada sucção, $\theta_{r}$ e $\theta_{s}$ representam as umidades volumétricas residuais e de saturação, respectivamente, e $\psi$ a sucção matricial; m, n, $\alpha$ são os parâmetros de ajuste.

\section{PREVISÃO DA RETENÇ̃̃O DE ÁGUA NO SOLO A PARTIR DO ESCALONAMENTO FRAC-} TAL Como mostrado anteriormente, a retenção de água no solo pode apresentar um comportamento peculiar devido ao escalonamento de poros encontrados em solos brasileiros. Este fato, observado por diversos pesquisadores (Oliveira et al. 2004, Lougon-Duarte et al. 2004, Feuerharmel et al. 2004, entre outros), foi em geral atribuído à estruturação do solo.

A maioria das funções baseadas em geometria fractal permite a previsão da curva de retenção numa faixa limitada em que o escalonamento de poros é possível apenas para baixos valores de sucção (próximas à saturação), sem levar em conta o comportamento particular de prováveis distribuições bimodais que podem ocorrer para sucções maiores às apresentadas nesses ensaios.

Um exemplo deste tipo de formulação foi o apresentado inicialmente por Alfaro Soto \& Vilar (2004). Esses autores apresentaram um modelo fractal para previsão da curva de retenção de água no solo, obtida considerando a distribuição dos poros (em vez das partículas do solo como concebido por Tyler \& Wheatcraft 1990), que é dada pela seguinte equação:

$$
\begin{array}{ll}
\mathrm{W}=\mathrm{w}_{\mathrm{s}}\left(\frac{\psi}{\psi_{\mathrm{b}}}\right)^{\mathrm{D}-3} & \left(\psi>\psi_{\mathrm{b}}\right) \\
\mathrm{W}=\mathrm{w}_{\mathrm{s}} & \left(\psi \leq \psi_{\mathrm{b}}\right)
\end{array}
$$

Nestas expressões, $\psi$ representa a sucção matricial para uma determinada umidade $\mathrm{w}, \psi_{\mathrm{b}}$ a pressão de entrada de ar, $\mathrm{w}_{\mathrm{s}}$ a umidade de saturação do solo e D a dimensão fractal decorrente do escalonamento fractal dos poros do solo; D é determinada a partir de alguns dados experimentais da curva de retenção ou mediante ensaios específicos (porosimetria, adsorção ou desorção isotérmica, entre outros).

Posteriormente, Alfaro Soto \& Vilar (2006) apontaram as dificuldades da equação (8) para previsão da retenção em maiores faixas de sucção e fora da mudança da trajetória do escalonamento dos poros, sendo necessário o uso de duas dimensões fractais, tal como mostrado na figura 4. Nestas figuras as curvas nomeadas com $\mathrm{D}_{1}$ e $\mathrm{D}_{2}$ mostram que o uso da equação (8), considerando duas dimensões fractais (produzido por dois escalonamentos de poros em um sistema heterogêneo), poderia ter duas pressões de entrada de ar ( $\psi_{\mathrm{b} 1}$ e $\left.\psi_{\mathrm{b} 2}\right)$. A primeira está associada com o início da dessaturação dos macroporos, enquanto a segunda pode estar associada, com a sucção correspondente ao teor de umidade residual. Nessas circunstâncias, a equação (8) é válida para valores de sucção entre a pressão de

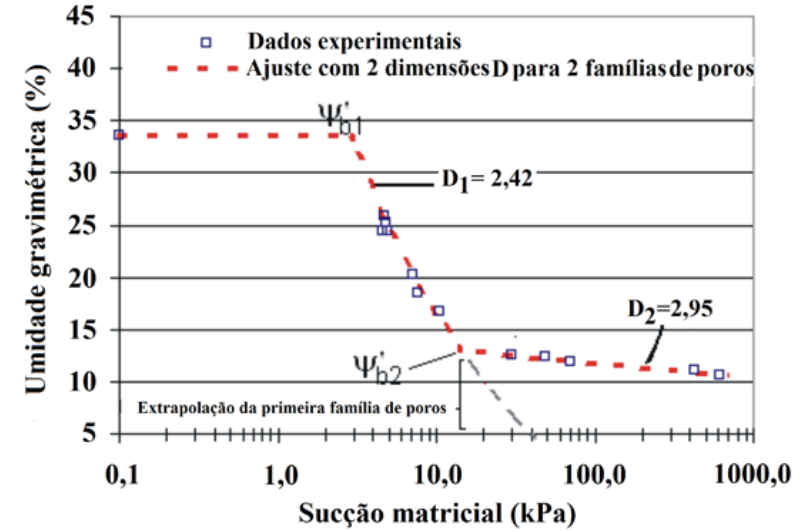

Figura 4 - Curva de retenção de uma argila arenosa com distribuição de poros bimodal com modelos fractal empregados para obtenção de dimensões $D_{1}$ e $D_{2}$ obtidas para cada família de poros (Alfaro Soto \& Vilar 2006).

entrada de ar $\left(\psi_{\mathrm{b}}\right)$ e aproximadamente a sucção correspondente à umidade residual.

Para levar em conta essa condição, a equação (8) foi modificada por meio de um artifício matemático que permite representar valores assintóticos próximos do teor de umidade residual. A modificação consiste inicialmente em isolar a dimensão fractal (D-3) da equação (8), obtendo-se:

$$
\mathrm{D}-3=\frac{\operatorname{Ln}(\mathrm{w})-\operatorname{Ln}\left(\mathrm{w}_{\mathrm{s}}\right)}{\operatorname{Ln}(\psi)-\operatorname{Ln}\left(\psi_{\mathrm{a}}^{\prime}\right)}
$$

Esta equação resulta equivalente à equação:

$$
\mathrm{D}-3=\frac{\operatorname{Ln}(\mathrm{w})-\operatorname{Ln}\left(\mathrm{w}_{\mathrm{r}}\right)}{\operatorname{Ln}(\psi)-\operatorname{Ln}\left(\psi_{\mathrm{i}}^{\prime}\right)}
$$

onde $\mathrm{w}_{\mathrm{r}}$ corresponde aproximadamente ao teor de umidade residual e $\psi^{\prime}{ }_{i}$ a sucção correspondente ao ponto de inflexão entre a primeira e a segunda família de distribuição de poros. Isto fica evidente na figura 5, que representa o escalonamento fractal da primeira família de poros.

Conseqüentemente, a partir da equação (10) podemos obter a expressão:

$$
\mathrm{W}=\mathrm{w}_{\mathrm{r}}\left(\frac{\psi}{\psi_{\mathrm{i}}^{\prime}}\right)^{\mathrm{D}-3}
$$

A partir de esta expressão foi necessário realizar uma adaptação para poder modelar a inflexão dos valores maiores que $\psi_{\mathrm{i}}$ e do comportamento assintótico em relação a $\mathrm{W}_{\mathrm{r}}$ compatível com a segunda família de poros. A expressão a seguir permite essa condição: 


$$
\lim _{\psi \rightarrow \infty} w_{r}+w_{r}\left(\frac{\psi}{\psi_{i}^{\prime}}\right)^{D-3}=w_{r}
$$

Nesta equação, quando o valor da sucção cresce indefinidamente a umidade se aproxima da residual. Assim, a equação modificada é expressa por:

$$
\begin{array}{ll}
\mathrm{w}=\mathrm{w}_{\mathrm{r}}\left(1+\left(\frac{\psi}{\psi_{\mathrm{i}}^{\prime}}\right)^{\mathrm{D}-3}\right) & \left(\psi>\psi_{\mathrm{b}}{ }^{\prime}\right) \\
\mathrm{w}=\mathrm{w}_{\mathrm{s}} & \left(\psi \leq \psi^{\prime}{ }_{\mathrm{b}}\right)
\end{array}
$$

A equação (13) permite melhor aproximação para maiores faixas de sucção e associa o comportamento assintótico numa única função.

\section{AVALIACÃO DA OCORRÊNCIA DO ESCALO-} NAMENTO FRACTAL Como mencionado anteriormente, o escalonamento fractal dos poros dos solos pode ser obtida diretamente por diversos ensaios. No entanto, pode ser determinado também a partir da curva de retenção da água do solo com um modelo destinado para esse fim, permitindo assim a determinação da sua dimensão fractal D, representativa do escalonamento.

Porém, antes desta avaliação, deve-se levar em consideração que solos brasileiros possuem particularidades, como características de poros multimodais que devem ser analisadas com a finalidade de estabelecer as limitações da ocorrência do escalonamento, bem como limitações de modelos para previsão da retenção de água no solo. Sendo assim, são apresentadas análises do comportamento multimodal e a previsão da retenção de água proveniente do sistema organizacional dos poros do solo.

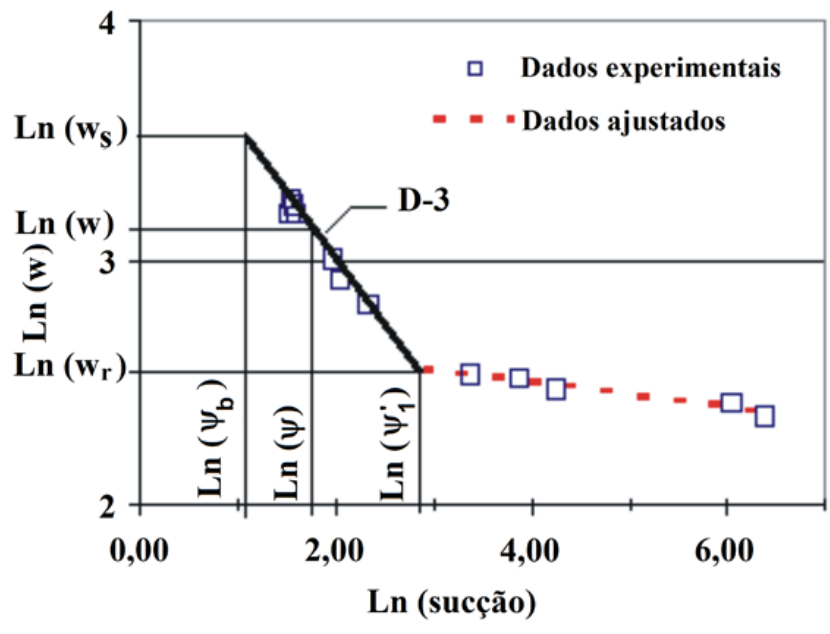

Figura 5 - Características do escalonamento fractal da figura 4, para a primeira família de poros.

\section{Avaliação do comportamento multimodal de alguns} solos inalterados $\mathrm{Na}$ avaliação do comportamento multimodal do solo foram utilizados resultados de seis tipos de solos. Os solos 1 e 2 são materiais inconsolidados provenientes da cidade de Santa Gertrudes, os solos 3 e 4 da cidade de Rio Claro, o solo 5, da cidade de Itirapina e o solo 6, da cidade de Caraguatatuba, todos do interior do estado de São Paulo. Na tabela 1, são sintetizadas algumas características geotécnicas destes materiais.

Como pode ser verificado nesta tabela, se observa que, foram empregados solos com textura argilosa e arenosa, com porosidades variáveis que podem ser ava-

\begin{tabular}{|c|c|c|c|c|c|c|c|}
\hline \multirow{2}{*}{ Parâmetros } & \multirow{2}{*}{ Und } & \multicolumn{6}{|c|}{ Solo } \\
\hline & & 1 & 2 & 3 & 4 & 5 & 6 \\
\hline Pedregulho & $\%$ & 0,2 & 3,2 & - & - & 4,4 & 2,7 \\
\hline Areia & $\%$ & 7,8 & 6,8 & 67,4 & 64,5 & 77,2 & 57,3 \\
\hline Silte & $\%$ & 16,0 & 21,0 & 24,0 & 23,3 & 1,4 & 34,0 \\
\hline Argila & $\%$ & 76,0 & 69,0 & 8,6 & 12,2 & 17,0 & 6,0 \\
\hline$\gamma$ & $\mathrm{kN} \cdot \mathrm{m}^{-3}$ & 27,1 & 27,3 & 25,8 & 26,1 & 26,7 & 26,6 \\
\hline$\gamma_{\mathrm{d}}$ & $\mathrm{kN} \cdot \mathrm{m}^{-3}$ & 15,6 & 12,2 & 15,0 & 14,1 & 13,8 & 12,0 \\
\hline e & - & 7,4 & 1,2 & 0,7 & 0,9 & 0,9 & 1,2 \\
\hline Textura & - & $\begin{array}{l}\text { Argila. } \\
\text { Siltosa }\end{array}$ & $\begin{array}{l}\text { Argila } \\
\text { Siltosa }\end{array}$ & $\begin{array}{c}\text { Areia } \\
\text { Siltosa }\end{array}$ & $\begin{array}{l}\text { Areia } \\
\text { Siltosa }\end{array}$ & $\begin{array}{c}\text { Areia } \\
\text { Argilosa }\end{array}$ & $\begin{array}{l}\text { Areia } \\
\text { Siltosa }\end{array}$ \\
\hline
\end{tabular}
liadas desde médias até elevadas (entre 0,72 até 1,24$)$.

Tabela 1 - Características geotécnicas dos solos estudados.

$\gamma$ e $\gamma_{\mathrm{d}}$ : peso específico dos sólidos e do solo respectivamente; e: índice de vazios. 
A análise consistiu em avaliar a distribuição do tamanho dos poros do solo a partir da expressão (7). Para esse objetivo, foram obtidas, para os seis solos estudados, as curvas de retenção dentro de uma ampla faixa de sucção, visando identificar a existência de mais de uma eventual distribuição de poros.

Na figura 6 têm-se os resultados experimentais da curva de retenção, ajustados (pelo modelo 6, porém em termos de umidade gravimétrica $\mathrm{w}$ ) por regressão não linear. Adicionalmente, a tabela 2 resume os parâmetros do ajuste.

Da figura 6 pode-se inferir que as curvas 1 e 2 seguem um comportamento típico de solos argilosos, de acordo com sua textura (Tab. 1); isto é, mostram maior capacidade de retenção em relação aos solos restantes, para o mesmo valor de sucção. Porém, a exceção corresponde ao solo 6 que, apesar da sua textura arenosa e elevada porosidade, mostrou uma elevada capacidade de retenção. Provavelmente, este fato possa ter explicação no tipo de argilomineral predominante na fração argila (não determinado no presente estudo).

$\mathrm{Na}$ mesma figura, resulta evidente a forma escalonada das curvas de retenção, sendo necessárias até três curvas de ajuste para o modelo de van Genuchten (1980), da equação (6). A esse respeito, a tabela 2 mos-

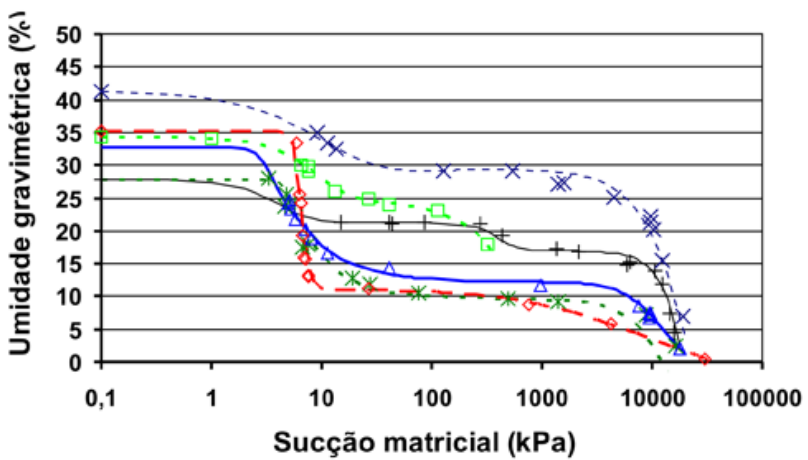

$$
\begin{aligned}
& \text { + Dados experimentais-Solo } 1 \text { Curva de ajuste-Solo } 1 \\
& \text { × Dados experimentais-Solo } 2 \text { - - - Curva de ajuste-Solo } 2 \\
& \text { Dados experimentais-Solo } 3 \text {... Curva de ajuste-Solo } 3 \\
& \text { Dados experimentais-Solo } 4-\text { Curva de ajuste-Solo } 4 \\
& \text { Dados experimentais-Solo } 5 \\
& \text { Dados experimentais-Solo } 6 \quad \text {. . Curva de ajuste-Solo } 6
\end{aligned}
$$

\begin{tabular}{|c|c|c|c|c|c|c|c|}
\hline Solo & Ajuste & $\mathrm{w}_{\mathrm{s}}$ & $\mathrm{w}_{\mathrm{r}}$ & $\alpha$ & $\mathrm{n}$ & $\mathrm{m}$ & $\mathrm{R}^{2}$ \\
\hline & & $(\%)$ & $(\%)$ & $\left(\mathrm{kPa}^{-1}\right)$ & & & \\
\hline \multirow[t]{3}{*}{1} & 1 & 27,8 & 21,2 & $1,80 \times 10^{-01}$ & 2,0005 & 1,9412 & 0,999 \\
\hline & 2 & 21,2 & 16,9 & $2,10 \times 10^{-03}$ & 4,1948 & 1,1789 & 0,991 \\
\hline & 3 & 16,9 & $-7,7$ & $3,20 \times 10^{-05}$ & 2,9042 & 0,9214 & 0,960 \\
\hline \multirow[t]{2}{*}{2} & 1 & 41,3 & 29,4 & $5,30 \times 10^{-03}$ & 0,9641 & 16,2236 & 0,994 \\
\hline & 2 & 29,4 & $-6,9$ & $2,90 \times 10^{-05}$ & 1,6523 & 0,8471 & 0,966 \\
\hline \multirow[t]{2}{*}{3} & 1 & 27,8 & 9,6 & $2,23 \times 10^{-01}$ & 10,867 & 0,1220 & 0,933 \\
\hline & 2 & 9,6 & $-27,5$ & $4,50 \times 10^{-05}$ & 2,1047 & 10,8670 & 0,991 \\
\hline \multirow[t]{2}{*}{4} & 1 & 32,7 & 12,2 & $3,47 \times 10^{-01}$ & 7,2359 & 0,1470 & 0,992 \\
\hline & 2 & 12,2 & $-5,6$ & $1,00 \times 10^{-04}$ & 2,5016 & 0,5073 & 0,953 \\
\hline \multirow[t]{2}{*}{5} & 1 & 35,2 & 11,0 & $1,55 \times 10^{-01}$ & 13,3837 & 0,8086 & 0,882 \\
\hline & 2 & 11,0 & $-93,0$ & $1,20 \times 10^{-03}$ & 1,0458 & 0,0281 & 1,000 \\
\hline \multirow[t]{2}{*}{6} & 1 & 34,4 & 24,8 & $2,13 \times 10^{-02}$ & 1,6821 & 15,7816 & 0,982 \\
\hline & 2 & 24,8 & $-11,5$ & $3,00 \times 10^{-04}$ & 1,3186 & 1,1853 & 0,994 \\
\hline
\end{tabular}

Figura 6 - Curvas de retenção para os solos (1 a 6).

tra os parâmetros obtidos e a aderência das curvas de ajuste aos dados experimentais, quando o coeficiente de determinação em todos os casos é próximo de um.

Tabela 2 - Parâmetros de ajuste por regressão.

$\mathrm{W}_{\mathrm{s}}, \mathrm{w}_{\mathrm{r}}$ : umidades gravimétricas de saturação e residual, respectivamente; $\mathrm{R}^{2}$ : coeficiente de determinação. 


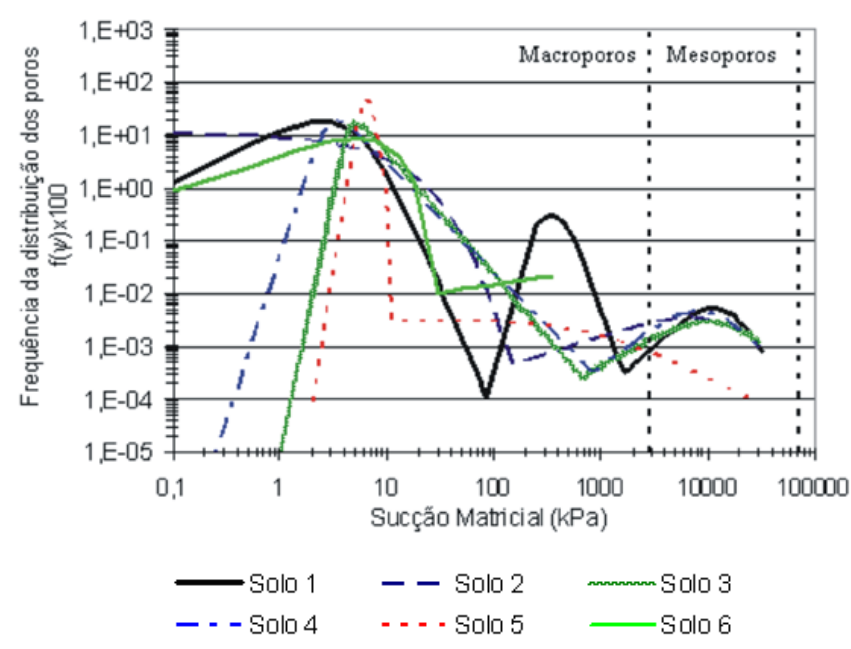

Figura 7 - Distribuição do tamanho dos poros dos solos (1 a 6).

Outro fato a ser observado nos parâmetros de ajuste são os resultados negativos para a umidade gravimétrica residual, produzidos pela extrapolação dos dados experimentais. Apesar destes valores não possuírem significado físico, eles foram utilizados para se obter o ajuste apenas nas faixas da curva em que se conta com dados experimentais.

Com a obtenção das curvas de retenção, procedeuse à determinação da distribuição do tamanho dos poros pela equação (7). A figura 7 ilustra esses resultados.

A figura 7 permite verificar que a maioria dos solos estudados possui distribuições de tamanho de poros bimodais, exceto o solo 1 , que se revelou trimodal. Os picos, inicial e final, identificam o início de escalonamentos de tamanho de poros que começam, aproximadamente, nas sucções correspondentes à pressão de entrada de ar e umidade residual, respectivamente. Já o pico intermediário, no solo 1 , representa um escalonamento intermediário.

Provavelmente, o comportamento multimodal dos poros deva-se à agregação das partículas minerais reunidas por agentes cimentantes (minerais de argila, matéria orgânica, óxidos de ferro e alumínio), formando partículas secundárias e poros maiores. Segundo Othmer et al. (1991), um sistema bimodal de poros pode estar composto por dois grupos: poros inter-agregados e intra-agregados. No primeiro, o movimento de água no solo é governado pelo potencial gravitacional e, no segundo, pelo potencial matricial.

Na figura 5, também foi incluída uma classificação proveniente de Web \& Orr (1997) em relação ao diâmetro dos poros, porém transformados em sucção matricial pela equação (5). A partir desta figura pode-se inferir que a agregação dos solos propiciou que a maioria dos solos avaliados tivesse uma e até duas distribuições na zona dos macroporos.
Previsão da retenção de água baseado no escalonamento fractal dos poros do solo Como já comentado, o modelo proposto por Alfaro Soto \& Vilar (2006) permite a previsão da retenção de água, inclusive para faixas de sucção superiores ao limite do primeiro escalonamento de poros do solo. No entanto, este modelo foi testado a partir de dados existentes na literatura, para solos não brasileiros.

Com a finalidade de testar o modelo apresentado por Alfaro Soto \& Vilar (2006) com solos brasileiros (cujas características são particulares), foram empregados diferentes tipos de solos indeformados e encontrados na literatura especializada. Os solos foram: argilas expansivas de Janga - PE (Melo et al. 1994), argila arenosa de Brasília - DF (Peixoto et al. 2001), solo arenoso, silto-argiloso e areia argilo-siltosa da região centro-oeste de SP (Santos \& Vilar 2004), solo siltoargiloso de Recife - PE (Jucá 1994), areia siltosa de Rio Claro - SP (Alfaro Soto \& Chang inédito) e areia silto-argilosa de Viçosa - MG (Custódio et al. 2004).

Para observar as limitações do modelo proposto por Alfaro Soto \& Vilar (2004), a equação (8) foi ajustada aos dados experimentais para o escalonamento entre aproximadamente a pressão de entrada de $\operatorname{ar}\left(\psi_{\mathrm{b}}\right)$ e a inflexão da curva. Por seu lado, a equação (13) foi ajustada para todos os dados experimentais. Ambos os resultados são apresentados nas figuras $8 \mathrm{a}$ à $8 \mathrm{j}$, mostrando a excelência de ajuste proveniente da segunda equação. A esse respeito, a tabela 3 apresenta os parâmetros dos ajustes obtidos por regressão não linear de ambos os ajustes. Nesta tabela pode-se observar que, para as duas formulações os coeficientes de determinação $\left(\mathrm{R}^{2}\right)$ são próximos a 1 mesmo para a equação (13), que considera, para a regressão, todos os dados experimentais. Por sua vez, a equação (8) precisaria de duas dimensões D para otimizar o ajuste.

A tabela 3 também mostra que em alguns casos (solos d, e, i) a pressão de entrada de ar $\left(\psi_{\mathrm{b}}\right)$ foi maior que o ponto de inflexão $\left(\psi_{i}\right)$ da curva da equação (13). Isto indica que esses solos provavelmente não possuem características fractais para a primeira família de poros e, nesse caso, a dimensão D passa a ser apenas um parâmetro de ajuste do modelo. Para os solos restantes se observa que $\psi^{\prime}$, foi maior que $\psi_{b}$, o que sugere que os solos possuem características fractais para toda a faixa de retenção medida (solos a, b, h) ou pelo menos parcialmente (solos restantes).

Visando avaliar se ambos os métodos (Equações 8 e 13) permitem valores médios aproximados da dimensão fractal, optou-se por uma análise estatística paramétrica (após verificar a normalidade da distribuição das amostras). Para a análise comparativa utilizouse o teste $t$, admitindo desvios padrão $(\sigma)$ das populações desconhecidos e diferentes, isto após verificar a igualdade de $\sigma$ com o teste $F$.

$\mathrm{O}$ teste $t$ acusou a igualdade dos valores médios das dimensões $\mathrm{D}$ provenientes de ambos modelos, para um nível de significância de 5\% (valor $\mathrm{P}=6,4 \%$ ).

Apesar da igualdade estatística do valor médio, observou-se que diferenças de $\mathrm{D}$, encontradas em de- 

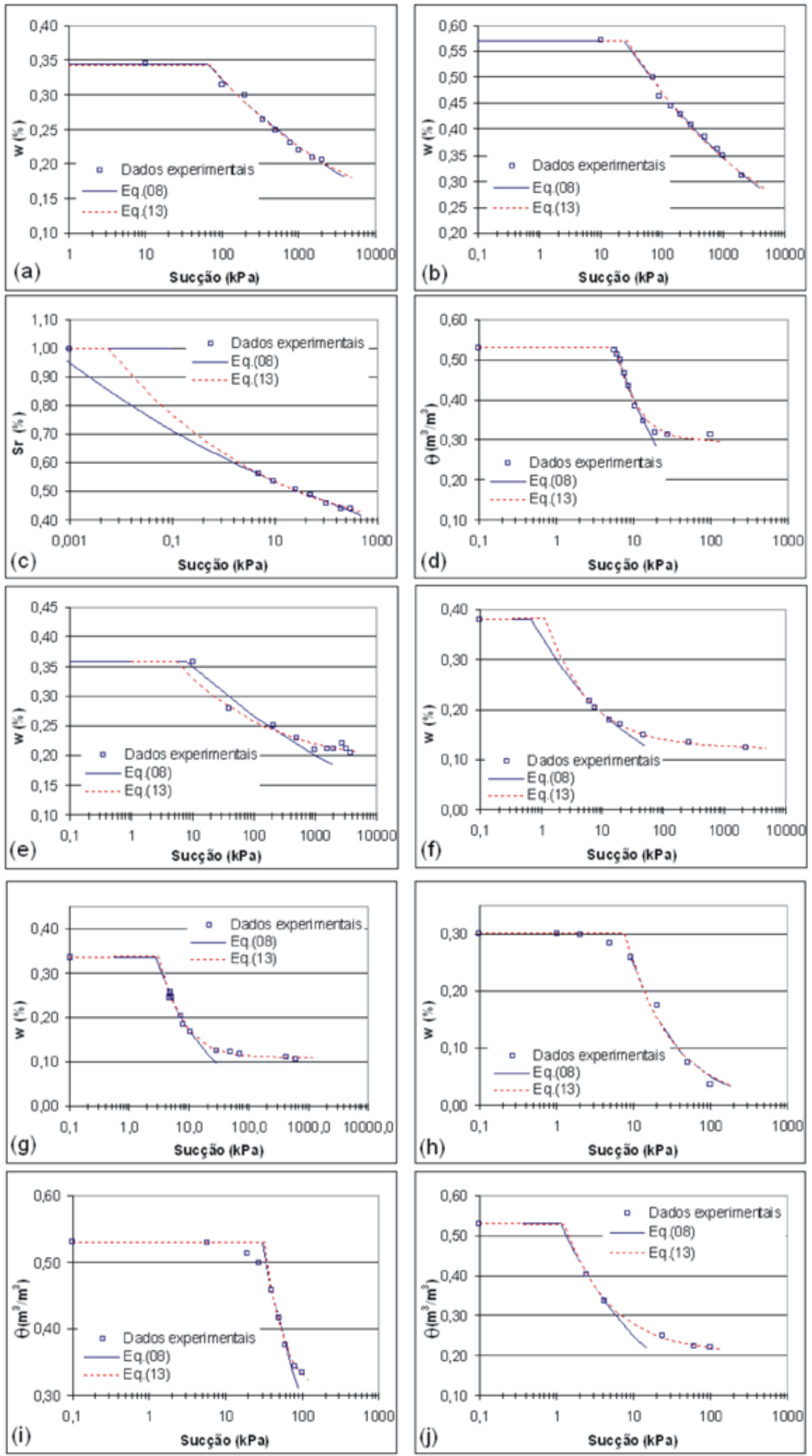

Figura 8 - Dados de curvas de retenção experimentais e ajustados pelas equações (8) e (13) nos solos (a) argiloso 1, (b) argiloso 2, (a e b provenientes de Melo et al. 1994), (c) argila arenosa (Peixoto et al. 2001), (d) silto-argiloso (Santos \& Vilar 2004), (e) silte argiloso (Jucá 1994), (f) areia siltosa (g) areia siltosa ( $f$ e g provenientes de Alfaro Soto \& Chang Inédito), (h) areia silto-argilosa (Custódio et al. 2004), (i) areia argilo-siltosa e (j) areia (i e j provenientes de Santos \& Vilar 2004). 
Tabela 3 - Parâmetros de ajuste por regressão para os modelos das equações (8) e (13).

\begin{tabular}{|c|c|c|c|c|c|c|c|c|c|}
\hline \multirow{3}{*}{ Fig. } & \multirow{3}{*}{ Tipo de Solo } & \multicolumn{8}{|c|}{ Parâmetros } \\
\hline & & \multicolumn{4}{|c|}{ Eq. (8) } & \multicolumn{4}{|c|}{ Eq.(13) } \\
\hline & & $\mathrm{w}_{\mathrm{s}}$ & $\psi_{\mathrm{b}}$ & $\mathrm{D}$ & $\mathrm{R}^{2}$ & $\mathrm{w}_{\mathrm{r}}$ & $\psi_{\mathrm{b}}^{\prime}$ & $\mathrm{D}$ & $\mathrm{R}^{2}$ \\
\hline $\mathrm{a}$ & Argiloso (Janga-PE) & 0,35 & 63,7 & 2,84 & 0,985 & 0,08 & 18507 & 2,78 & 0,980 \\
\hline $\mathrm{b}$ & Argiloso (Janga-PE) & 0,57 & 24,5 & 2,87 & 0,994 & 0,11 & 79082 & 2,82 & 0,990 \\
\hline $\mathrm{c}$ & Argila arenosa (Brasília-DF) & 1,00 & 0,004 & 2,94 & 0,992 & 0,27 & 11,6 & 2,87 & 0,993 \\
\hline d & Silte argil.(Centro Oeste-SP) & 0,53 & 5,7 & 2,50 & 0,992 & 0,29 & 5,1 & 1,44 & 0,983 \\
\hline e & Silte argiloso (Recife - PE) & 0,36 & 7,9 & 2,88 & 0,896 & 0,18 & 5,1 & 2,71 & 0,940 \\
\hline $\mathrm{f}$ & Areia siltosa (Rio Claro - SP) & 0,38 & 0,7 & 2,75 & 0,993 & 0,12 & 4,0 & 2,39 & 0,998 \\
\hline $\mathrm{g}$ & Areia siltosa (Rio Claro - SP) & 0,34 & 2,8 & 2,46 & 0,964 & 0,11 & 6,0 & 1,88 & 0,992 \\
\hline $\mathrm{h}$ & Areia silto-argilosa (Visoça-MG) & 0,30 & 7,6 & 2,31 & 0,975 & 0,003 & 4515 & 2,29 & 0,974 \\
\hline $\mathrm{i}$ & Areia arg.-siltosa (Centro Oeste-SP) & 0,53 & 29,7 & 2,51 & 0,993 & 0,30 & 28,1 & 1,32 & 0,991 \\
\hline $\mathrm{j}$ & Areia (Centro Oeste - SP) & 0,53 & 1,1 & 2,66 & 1,000 & 0,21 & 2,3 & 2,28 & 0,998 \\
\hline
\end{tabular}

terminadas amostras (principalmente nos solos d, g, i), podem ser ocasionadas devido ao ajuste pela Equação (13), que leva em consideração, para o calculo, uma faixa do escalonamento mais extensa em relação à obtida pela Equação 8, que está sujeita apenas aos valores experimentais. Embora a Equação (13) permita uma maior abrangência para o cálculo de $\mathrm{D}$, verifica-se que este fato modifica sensivelmente seus resultados, comprometendo assim o estabelecimento de valores característicos de D para cada tipo de solo.

Sendo assim um próximo passo será avaliar a variabilidade de $\mathrm{D}$ quando relacionada com algumas propriedades ou índices do solo. O único dado adicional disponível para os solos aqui analisados foi sua textura, e devido à pequena quantidade de exemplos testados, não foi possível encontrar alguma tendência entre essas duas variáveis.

CONCLUSÕES Este trabalho apresenta os comportamentos de escalonamentos de poros avaliados mediante curvas de retenção, em que suas distribui- ções mostram-se multimodais devido a características peculiares, como já apontadas por diversos autores em alguns solos brasileiros.

Observou-se que, em geral os picos modais dos poros estão relacionados com seus escalonamentos e que podem ocorrem em toda a faixa da distribuição dos seus tamanhos. Assim, foram detectados em alguns solos até dois picos modais na faixa de macroporos e um na de mesoporos. A presença de diferentes escalonamentos nestes solos dificulta a utilização de modelos tradicionais, baseados na geometria fractal para a previsão da retenção de água no solo, limitando sua previsão para faixas pequenas de sucção (entre 10 e $50 \mathrm{kPa}$ de sucção segundo o observado neste trabalho).

Por sua vez, o modelo formulado por Alfaro Soto \& Vilar (2006) mostrou-se adequado para retratar a curva de retenção de água em diferentes tipos de solos indeformados brasileiros, ajustando-se satisfatoriamente aos dados experimentais em uma maior faixa de sucção. Adicionalmente, foi capaz de reproduzir a curva de retenção utilizando apenas uma dimensão fractal.

\section{Referências}

Alfaro Soto M.A. 1999. Estudo da condutividade hidráulica em solos não saturados. Dissertação de Mestrado, Escola de Engenharia de São Carlos, Universidade de São Paulo, 121p.

Alfaro Soto M.A. 2004a. Comparação entre métodos de imposição e de controle de sucção em ensaios com solos não saturados. Tese de doutoramento, Escola de Engenharia de São Carlos, Universidade de São Paulo, 210p.

Alfaro Soto M. A. \& Vilar O.M. 2004b. Geometria fractal na deter- 
minação da curva de retenção de água no solo. In: Simpósio Brasileiro de Solos Não Saturados, 5, Atas, v.1, p. 203-208.

Alfaro Soto M.A. \& Vilar O.M. 2006. Evaluation of a Pore Fractal Model for the Prediction of Soil Water Retention Curve. In: International Conference on Unsaturated Soils, 4, ArizonaUSA, Proceedings, ASCE Geotechnical Special Publication, 147(2):2441-2452.

Buckingham E. 1907. Studies on the movement of soil moisture. U.S. Dept. Agr. Bur. Soils, Bul., 38.

Feuerharmel C., Gehling W.Y.Y., Bica A.V.D., Pereira A. 2004. Determinação das curvas características de solos coluvionares pelo uso combinado da placa de sucção e método do papel filtro. In: Simpósio Brasileiro de Solos Não Saturados, 5, São Carlos, atas, v.1, p. 243-248.

Gimenez D., Perfect E., Rawis W.J., Pachepsky Y.A. 1997. Fractal models for predicting soil hydraulic properties: a review. Engineering Geology, 48:161-183.

Jucá J.F.T 1994. The prediction of total heave using laboratory and field tests data. In: Simpósio Brasileiro de Solos Não Saturados, 2, Recife, Atas, v.1, p. 89-97.

Libardi P.L. 1995. Dinâmica da Água no Solo. Edição do Autor, ESALQ, Depto. de Fisica e Metereologia, Piracicaba, $497 \mathrm{p}$.

Lougon-Duarte A.P., de Campos T.M.P., Araruna J.T. 2004. Efeitos da temperatura na relação umidade-sucção de dois solos. In: Simpósio Brasileiro de Solos Não Saturados, 5, São Carlos, Atas, v.1, p. 235-242.

Mallants D., Tseng P.H., Toride N., Timmerman A., Feyen J. 1997. Evaluation of multimodal hydraulic functions in characterizing a heterogeneous field soil. Journal of hydrology, 95:172-199.

Mello R.M., Bastos E.G., Jucá J.F.T. 1994. Propriedades de fluxo de solos expansivos não saturados. In: Simpósio Brasileiro de Solos Não Saturados, 2, Recife, Atas, v.1, p. 135-140.

Oliveira D.R., Gitirana Jr. G.F.N., Pereira J.H.F 2004. Influência das propriedades hidráulicas na evaporação de solos colapsíveis não saturados. In: Simpósio Brasileiro de Solos Não Saturados,
5, São Carlos, Atas, v.1, p. 161-167.

Othmer H., Diekkruger B., Kutilec M. 1991. Bimodal porosity and unsaturated hydraulic conductivity. Soil Science, 52:139-150.

Pachepsky Y., Crawford J.C., Rawls W.J. 2000. Fractals in soil science. Elsevier Healt Sciences, p. 302.

Peixoto R.J., Feitosa J.H.P., Gitirana G.F.N., Cunha R.P., Carvalho J.C. 2001. Superfície de estado do solo poroso colapsível do Distrito Federal. In: Simpósio Brasileiro de Solos Não Saturados, 4, Porto Alegre, Atas, v.1, p. 93-109.

Santos C.R. \& Vilar O.M. 2004. Análise paramétrica da estabilidade de taludes em solos não saturados: a influência no tipo de solo. In: Simpósio Brasileiro de Solos Não Saturados, 5, São Carlos, Atas, v.1, p. 181-188.

Stoicescu J.T., Haug M.D., Fredlund D.G. 1996. In: Canadian Geotechnical Conference, $49^{\text {th }}$, St. Johns, Newfoundland., Proceedings, vol. 2. p. 721-728.

Turcotte L.D. 1992. Fractals and caos in geology and geophysics. New York, Cambridge University Press, p. 221.

Tyler W.S. \& Wheatcraft S.W. 1990. Fractal processes in soil water retention. Water Resources Research, 26(5):1047-1990.

van Genuchten M.T. 1980. A closed-form equation for predicting the hydraulic conductivity of unsaturated soils. Soil Sci. Soc. Am.J., 44(5):892-898.

Webb P.A. \& Orr C. 1997. Analytical Methods in Fine Particle Technology. Micromeritics Instrument Corporation, NorcrossGA, USA, 301p.

Xu Y.F., Sun D.A., Wang J.H. 2002. Fractal model for water flow through unsaturated soils. In: Int. Conf. on Unsaturated Soils, 3, Recife, Brazil, Balkema, Proceedings, Vol. 1, p. 21-26,

Manuscrito ID 9653

Submissão em 30 de outubro de 2007 Aceite em 20 de junho de 2008 Sistema eletrônica de submissão 